15. Association between glycated hemoglobin $(\mathrm{HbA} 1 \mathrm{c})$ and the lipid profile in patients with type 2 diabetes mellitus at a tertiary care hospital: a retrospective study / S.H. Alzahrani, M. Baig, M.M. Aashi [et al.] // Diabetes Metab Syndr Obes. 2019; 12: 1639-1644. doi: 10.2147/DMSO.S222271.

16. Radin M.S. Pitfalls in Hemoglobin A1c Measurement: When Results may be Misleading / M.S Radin // J Gen Intern Med. 2014; 29(2): 388-394. doi: 10.1007/s11606-013-2595-X.

17. Increased plasma malondialdehyde and fructosamine in iron deficiency anemia: effect of treatment / R.C. Sundaram, N. Selvaraj, G. Vijayan [et al.] // Biomed Pharmacother 2007; 61: 682-5. doi: 10.1016/j.biopha.2007.06.013/

18. Welsh K.J. Role of glycated proteins in the diagnosis and management of diabetes: research gaps and future directions / K.J. Welsh, M.S. Kirkman, D.B. Sacks // Diabetes Care 2016; 39: 1299-306. doi: 10.2337/dc15-2727.

19. The Relationship Between Alcohol Consumption and Glycemic Control Among Patients with Diabetes: The Kaiser Permanente Northern California Diabetes Registry / A.T. Ahmed, A.J. Karter, E.M. Warton [et al.] // J Gen Intern Med. 2008; 23(3): 275-282. doi: 10.1007/s11606-007-0502-z.

20. Hong J.W. Association between Alcohol Intake and Hemoglobin A1c in the Korean Adults: The 2011-2013 Korea National Health and Nutrition
Examination Survey / J.W. Hong, J.H. Noh, D.J. Kim // Plos one 2016; 11(11): e0167210. doi.org/10.1371/journal.pone.0167210.

21. Serum bilirubin is significantly associated with Hba1c in type 2 diabetic subjects / T. Farasat, S. Sharif, F. Manzoor et al. // Endocrinol Metab Int J. 2017; 5(6): 338-341. doi: 10.15406/emij.2017.05.00142.

22. Differential Effects of Azithromycin, Doxycycline, and Cotrimoxazole in Ingested Blood on the Vectorial Capacity of Malaria Mosquitoes / M. Gendrin, R.S. Yerbanga, J.B. Ouedraogo [et al.] // Open Forum Infectious Diseases 2016; 3(2): ofw074. doi.org/10.1093/ofid/ofw074.

23. Cappellini M.D. Glucose-6-phosphate dehydrogenase deficiency / M.D. Cappellini, G. Fiorelli // Lancet. 2008; 371(9606): 64-74. doi: 10.1016/S0140-6736(08)60073-2.

24. Aspirin-mediated acetylation of haemoglobin increases in presence of high glucose concentration and decreases protein glycation / F. Finamore, F. PriegoCapote, S. Nolli [et al.] // EuPA Open Proteomics 2015; 8: 116-127. doi.org/10.1016/j.euprot.2015.04.003.

25. Kotb A. Effect of Vitamin C on Blood Glucose and Glycosylated Hemoglobin in Type II Diabetes Mellitus / A. Kotb, K.M. Al Azzam // World Journal of Analytical Chemistry 2015; 3(1A): 6-8. doi: 10.12691/wjac-3-1A-2.

\title{
THE FREQGUENCY OF CRITICAL INCIDENTS AT PERIOPERATIVE PERIOD IN SMOKING PATIENTS
}

\author{
DOI: $10.31618 /$ ESU.2413-9335.2020.3.73.668 \\ V.D. Slepushkin,* M.C. Musaeva, \\ G.G. Bestaev, P. V. Musaeva \\ North-Ossetian State Medical Academ, \\ Vladikavkaz 362025, Russia
}

\begin{abstract}
A prospective, randomized, open research was performed, including 114 patients who had laparoscopic cholecystectomy: 1 group - non-smokers (57 people), 2 group - smokers (57 people).

Among smokers, both during the operation and within the near postoperative period (up to 120 minutes), the number of critical incidents increases, connected with the following systems: respiratory system, blood circulation system, and, especially, nervous system: motor excitation, fever, muscular tremors, the feeling of air lack.

Research Results allow to recommend to the anesthetist to pay attention to the smoking experience, the quantity of smoked cigarettes per day and to be wary concerning the occurrence of possible critical incidents.

Keywords: smoking, critical incidents, perioperative period

\section{1.Введение}

В мире ежегодно регистрируется 5,4 миллиона смертей, связанных с курением [1]. Курение увеличивает риск неблагоприятных послеоперационных исходов, особенно сердечных и легочных осложнений $[2,3]$. У курильщиков преобладают легочные проблемы, учитывая, что курение ухудшает транспорт слизи, провоцирует гиперплазию бокаловидных клеток, гиперсекрецию слизи [4], ухудшает функцию легочных макрофагов [5], увеличивает реактивность бронхов путем стимуляции воспаления дыхательных путей [6]. Достаточно хорошо установлено, что курение способствует развитию сердечно-сосудистых заболеваний,

однако взаимосвязь между курением и периоперационными сердечно-сосудистыми осложнениями остается спорной [7]. В большинстве исследований не четко идентифицирован предоперационный статус курения в качестве независимого фактора сердечных событий после выполнения некардиальных хирургических вмешательств [2, 8, 9]. Одним из способов выявления влияния курения на возникновение сердечно- сосудистых и легочных осложнений в интраоперационном периоде является регистрация критических инцидентов $[10,11]$. Под критическим инцидентом понимается событие при проведении анестезиологического пособия, которое при
\end{abstract}


отсутствии вмешательства может привести к нежелательным последствиям [11].

Цель исследования: определить частоту возникновения критических инцидентов со стороны сердечно-сосудистой и легочной систем у курящих и некурящих пациентов в периоперационном периоде.

\section{2.Материал и методы}

Выполнено контролируемое проспективное рандомизированное клиническое исследование. План рандомизации был сгенерирован по закону случайных чисел на сайте www.randomization.com (seed 7481). Обследовано 114 пациентов с хроническим калькулезным холециститом, которым будет выполнена лапароскопическая холецистэктомия в условиях тотальной внутривенной анестезии на основе дипривана.

Критерии включения:

- информированное согласие пациента на участие в исследовании; холецистэктомии;

- физический статус класса $\mathrm{ASA}_{\text {и }} \mathrm{ASA}_{\mathrm{II}}$;

- возраст 38-52 года.

Критерии исключения:

- отказ больного от участия в исследовании (отсутствие информированного согласия);

- физический статус класса ASA

- переход на открытую операцию;

- наличие в анамнезе поливалентной аллергии, сахарного диабета, декомпенсированных нарушений со стороны сердечно-легочной системы.

Пациенты разделены на две группы: 1-я некурящие (57 пациентов); 2-я - курящие в день не менее 20 сигарет в течение не менее 10 лет (57 пациентов). Демографические данные, информация об индексе массы тела и физическом статусе пациентов представлены в таблице 1. Длительность анестезии в обеих группах колебалась в пределах 54-72 минуты.

Таблица 1

Демографические данные пациентов, индекс массы тела и физический статус по ASA

\begin{tabular}{|c|c|c|c|c|c|c|}
\hline Группы & Количество & Пол & Возраст & Индекс массы тела & ASA I & ASA II \\
\hline 1 & 46 & $\mathrm{M}$ & $45,3+5,2$ & $25,8+4,7$ & 39 & 7 \\
\hline & 11 & Ж & $47,4 \pm 5,1$ & $26,8 \pm 4,8$ & 8 & 3 \\
\hline & 45 & $\mathrm{M}$ & $44,4 \underline{ \pm} 5,6$ & $24,6 \pm+4,5$ & 35 & 10 \\
\hline Всего & 12 & Ж & $46,7 \underline{7}+1$ & $26,1 \pm 5,0$ & 8 & 4 \\
\hline
\end{tabular}

Примечание: данные представлены в виде $\mathrm{M} \pm$ м. Во всех случаях $\mathrm{P}>0,05$.

Протокол стандартной ТВА (для обеих групп):

-Премедикация : димедрол 1\% - 1,0 мл внутримышечно;

-Преоксигенация :1-2 минуты;

-Индукция:пропофол 2 мг/кг, фентанил 2,04,0 мкг/кг, круарон 0,6 мг/кг;

-Поддержание анестезии:фентанил +пропофол 2-5 мг/кг в час;

-Миоплегия: круарон0,15 мг/кг при появлении первого ответа на ТOF - стимуляцию;

•Объем инфузии: кристаллоиды 5-7 мл/кг · ч.

Методы исследования

•ЭКГ, ЧСС, неинвазивное измерение АД, капнометрия;

•BIS-мониторинг глубины угнетения сознания;

-TOF - Watch - акцелеромиографический мониторинг количественной нейромышечной функции.
Рассчитывали частоту критических инцидентов (ЧКИ) и индекс частоты критических инцидентов (ИЧКИ) [12].

Регистрацию критических инцидентов (КИ) проводили в середине проведения анестезии и в ближайшем послеоперационном (30-40 мин) периоде.

Результаты представлены как среднее (M), среднего отклонения от среднего арифметического (м). Статистическую обработку полученных результатов выполняли с помощью пакета программ STATISTICA 7,0. Статистически достоверным считали $\mathrm{p}<0,05$. При статистическом анализе ЧКИ использован метод четырехпольной таблицы сопряженных частот [13].

\section{3.Результаты и обсуждение}

Количественный анализ критических инцидентов и частота критических инцидентов у пациентов во время и после анестезии приведен в таблице 2.

Таблица 2

Распределение критических инцидентов в группах во время и после анестезии

\begin{tabular}{|c|c|c|c|}
\hline Этапы регистрации & 1 группа КИ (ЧКИ) & 2 группа КИ (ЧКИ) & Критерии \\
\hline Во время анестезии & 5 (ЧКИ=0,09) & 17 (ЧКИ=0,30) & $\mathrm{P}<0,05$ \\
\hline После анестезии & 9 (ЧКИ $=0,16)$ & $39($ ЧКИ=0,71) & $\mathrm{P}<0,001$ \\
\hline Всего & 14 (ЧКИ $=0,25)$ & $56($ ЧКИ=0,98) & $\mathrm{P}<0,001$ \\
\hline
\end{tabular}

Во время проведения анестезии ЧКИ у пациентов 2-й группы выше, чем у пациентов 1-й группы в 3,4 раза, тогда как после окончания анестезии ЧКИ у пациентов 2-й группы выше в 4,3 раза по сравнению с пациентами 1-й группы.

Качественный состав критических инцидентов у пациентов обеих групп во время проведения 
анестезиологического пособия и после его окончания представлен в таблицах 3 и 4.

Таблица 3

Качественный состав критических инцидентов (КИ) во время проведения анестезии \begin{tabular}{l|l|l|l|} 
Инцидент & 1 группа ( 57 пациентов) & 2 группа ( 57 пациентов) & Статистическая значимость \\
\hline
\end{tabular}

КИ, связанные с системой дыхания

\begin{tabular}{|c|c|c|c|}
\hline Ларингоспазм & - & 1 & \\
\hline Всего & 0 & 1 (ЧКИ=0,01) & \\
\hline
\end{tabular}

КИ, связанные с системой кровообращения

\begin{tabular}{|c|c|c|c|}
\hline $\begin{array}{c}\text { Тахикардия } \\
\text { ЧСС }>120 \\
\text { уд в мин } \\
\end{array}$ & 1 & 3 & \\
\hline $\begin{array}{c}\text { Брадикардия } \\
\text { ЧСС <50 } \\
\text { уд в мин } \\
\end{array}$ & 1 & 3 & \\
\hline $\begin{array}{c}\text { Гипертензия } \\
\text { АДсист }>30 \% \\
\text { от величины после } \\
\text { вводного наркоза }\end{array}$ & 2 & 3 & \\
\hline $\begin{array}{c}\text { Гипотензия } \\
\text { АДсист } 30 \% \\
\text { от величины после } \\
\text { вводного наркоза }\end{array}$ & 1 & 2 & \\
\hline $\begin{array}{c}\text { Депрессия или } \\
\text { подъем сегмента } \\
\text { ST } \\
\end{array}$ & - & 2 & \\
\hline Аритмия & 1 & 2 & \\
\hline Всего & 5 (ЧКИ=0,09) & 13 (ЧКИ=0,23) & $\mathrm{P}<0,05$ \\
\hline
\end{tabular}

КИ, связанные с нервной системой

\begin{tabular}{|c|c|c|c|}
\hline $\begin{array}{l}\text { Двигательное } \\
\text { возбуждение }\end{array}$ & - & 2 & \\
\hline $\begin{array}{l}\text { Синдром озноба и } \\
\text { мышечной дрожи }\end{array}$ & - & 1 & \\
\hline Всего & 0 & $3($ ЧКИ=0,06) & \\
\hline ИТОГО & $5($ ЧКИ=0,09) & $17($ ЧКИ=0,30) & $\mathrm{P}<0,05$ \\
\hline
\end{tabular}

Среди КИ, связанных с системой дыхания, у курящих пациентов (2-я группа) в одном случае во время вводного наркоза зафиксирован ларингоспазм, несмотря на то, что глубина анестезии по БИС-монитору составляла $35 \%$, а на ТОФ-мониторе регистрировалось ТОF - 0 ответов. Ларингоспазм ликвидирован дополнительным болюсным введением 50 мг дипривана и 30 мг круарона.

Число КИ, связанные с системой кровообращения, статистически достоверно чаще фиксировались у пациентов 2-й группы.

У пациентов обеих групп в процессе проведения анестезиологического пособия глубина утраты сознания по показателям БИС-монитора составляла 50-45\%, что мы считаем наиболее оптимальной величиной [14]. Для достижения указанных значений БИС-мониторинга значения доз дипривана составили одинаковые величины в обеих группах пациентов. У некурящих пациентов для адекватного обезболивания расход фентанила

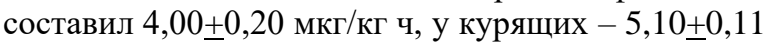
мкг/кг ч $(\mathrm{P}<0,05)$. Адекватность обезболивания оценивали по возрастанию ЧСС более чем на $20 \%$ и по снижению величины реоплетизмограммы более чем на $20 \%$ от величин, регистрируемых при вводном наркозе. Расход круарона в группе некурящих пациентов составил $0,48 \pm 0,03$ мГ/кГ ч, в группе курящих - 0,61 $\pm 0,03$ мг/кг ч $(\mathrm{P}<0,05)$.

У пациентов 2-й группы непосредственно перед экстубацией регистрировали синдром двигательного возбуждения и синдром мышечной дрожи. При этом индекс ТОFсоставлял 0.8-0.9. 
Таблица 4

Качественный состав критических инцидентов (КИ) после окончания анестезии

\begin{tabular}{|c|c|c|c|}
\hline Инцидент & $\begin{array}{c}1 \text { группа } \\
(57 \text { пациентов })\end{array}$ & $\begin{array}{c}2 \text { группа } \\
(57 \text { пациентов })\end{array}$ & $\begin{array}{c}\text { Статистическая } \\
\text { значимость }\end{array}$ \\
\hline
\end{tabular}

\begin{tabular}{|c|c|c|c|}
\hline \multicolumn{4}{|c|}{ КИ, связанные с системой дыхания } \\
\hline Ларингоспазм & - & 2 & \\
\hline Стридор & 1 & 2 & \\
\hline $\begin{array}{c}\text { Умеренная гипоксемия } \\
\mathrm{SpO}_{2}<90>80 \% \\
\end{array}$ & 2 & 3 & \\
\hline $\begin{array}{c}\text { Критическая гипоксемия } \\
\mathrm{SpO}_{2}<80 \%\end{array}$ & - & 1 & \\
\hline $\begin{array}{c}\text { Критическая гиперкапния } \\
\mathrm{E}_{\mathrm{C}} \mathrm{CO}_{2}>55 \text { мм рт.ст. }\end{array}$ & 1 & 3 & \\
\hline $\begin{array}{l}\text { Остановка дыхания } \\
\text { более чем на } 45 \text { сек }\end{array}$ & - & 1 & \\
\hline Всего & 4 (ЧКИ $=0,09)$ & $12($ ЧКИ $=0,21)$ & $\mathrm{P}<0,05$ \\
\hline
\end{tabular}

КИ, связанные с системой кровообращения

\begin{tabular}{|c|c|c|c|}
\hline Тахикардия & 1 & 4 & \\
\hline Брадикардия & - & 2 & \\
\hline Гипертензия & 1 & 5 & \\
\hline Гипотензия & - & - & \\
\hline Депрессия или подъем ST & - & 3 & \\
\hline Аритмия & 1 & 4 & $\mathrm{P}<0,001$ \\
\hline Всего & 3 (ЧКИ=0,04) & $18($ ЧКИ $=0,30)$ & \\
\hline
\end{tabular}

КИ, связанные с нервной системой

\begin{tabular}{|c|c|c|c|}
\hline Двигательное возбуждение & - & 3 & \\
\hline $\begin{array}{c}\text { Синдром озноба и мышечной } \\
\text { дрожи }\end{array}$ & 1 & 3 & \\
\hline Чувство нехватки воздуха & 1 & 3 & $\mathrm{P}<0,01$ \\
\hline Всего & $2($ ЧКИ $=0,04)$ & $9($ ЧКИ=0,16) & $\mathrm{P}<0,001$ \\
\hline ИТОГО & 9 (ЧКИ $=0,16)$ & $39($ ЧКИ $=0,71)$ & \\
\hline
\end{tabular}

Суммарное значение ЧКИ в обеих группах пациентов в послеоперационном периоде примерно соответствует данным других авторов, которые проводили ТВА у пациентов при выполнении лапароскопической холецистэктомии [15].

В ближайшем послеоперационном периоде у курящих пациентов в 3,0 раза чаще регистрировались КИ со стороны системы дыхания, в 6 раз чаще КИ, связанные с системой кровообращения, и в 4,5 раз КИ, связанные с нервной системой. В общей сложности, частота

возникновения КИ и, соответственно ЧКИ, у курящих пациентов была в 4,3 выше, чем у не курящих.

Экстубацию всех пациентов проводили при показателях БИС-монитора в пределах 88-92\%. Индекс TOF от 0.9 считали отражением адекватного восстановления нейромышечной функции.

Результаты о времени от момента окончания введения гипнотика и миорелаксанта до экстубации представлено в таблице 5.

Таблица 5

Время от окончания инфузии дипривана и последнего введения миорелаксанта до экстубации

\begin{tabular}{|c|c|c|}
\hline $\begin{array}{c}\text { Группы } \\
\text { пациентов }\end{array}$ & $\begin{array}{c}\text { Время в мин от отключения дипривана } \\
\text { до экстубации }\end{array}$ & $\begin{array}{c}\text { Время в мин от последнего введения } \\
\text { миорелаксанта до экстубации }\end{array}$ \\
\hline 1 & $6,30 \pm 1,30$ & $26,80 \pm 3,90$ \\
\hline 2 & $6,50 \pm 1,40$ & $41,60 \pm 3,10$ \\
\hline $\mathrm{P}$ & $>0,05$ & $<0,05$ \\
\hline
\end{tabular}

Диприван не влияет на время экстубации и, скорее всего, не играет существенной роли в возникновении критических инцидентов, отмечающихся у пациентов в послеоперационном периоде. Следовательно, компонентами ТВА, которые могли бы провоцировать возникновение критических инцидентов, могут быть опиоидные аналгетики и миорелаксанты. Известно, что у курильщиков отмечается значительная секвестрация суфентанила и фентанила легкими [16]. Для полноценной анальгезии курильщикам необходимо введение повышенных доз опиоидов [ 17], а для полноценной миорелаксации им требуется увеличение дозы рокурония примерно на 25\% по сравнению с не курящими пациентами [18]. Аналогичные результаты приведены в настоящем исследовании: у курящих пациентов дозы фентанила возрастали на $28 \%$, что может быть следствием снижения чувствительности мюопиатных рецепторов продуктами, содержащимися в табачном дыме [16]. Дозы использованного рокурония повышались на 27\%. У курящих 
пациентов также увеличивается и продолжительность действия векурония и рокурония $[19,20]$, что отмечено и в настоящем исследовании. Это может объясняться изменением их метаболизма в печени [21]. Таким образом, кумуляция повышенных доз опиоидов и миорелаксантов могут быть факторами возникновения критических инцидентов легочной системы. Ухудшение транспорта слизи при одновременной гиперсекреции слизи (мукоцилиарный клиренс) [4] наряду с остаточной миорелаксацией [22] провоцирует более частые эпизоды гипоксемии и гиперкапнии у курящих лиц, что регистрировалось в настоящем исследовании. Повышение у курильщиков реактивности бронхов наряду с остаточной миорелаксацией может провоцировать возрастание частоты ларингоспазма Курение также является независимым фактором увеличения инцидентов со стороны сердечнососудистой и нервной систем как во время, так и после хирургических вмешательств в некардиальной хирургии [22].

\section{4.Заключение}

Возрастание у курящих лиц частоты критических инцидентов со стороны легочной, сердечно-сосудистой и нервной систем во время и после проведения хирургического вмешательства может быть следствием суммирующего действия компонентов, входящих в состав сигаретного дыма, необходимости в повышенном количестве опиоидов и миорелаксантов, их кумуляции и изменением метаболизма в печени.

\section{References}

[1] P.K.Mandal ,D. Schifilliti ,F Mafrica , V.Fodale : Inhaled anesthesia and cognitive performance. Drugs Today (Barc), 2009, hh. 45:47. Doi.org/10.1358/dot.2009.45.1.1315075

[2] N.Al-Sarraf ,L Thalib , A. Hughes et al., “ Effect of smoking on short-term outcome of patients undergoing coronary artery bypass surgery", Ann Thorac Surg., vol.86, pp. 517-523, 2008; .Doi.org/10.1016/j.athoracsur.2008.03.070

[3] P.Rock , P.B. Rich “ Postoperative pulmonary complications" Curr Opin Anaesthesiol., vol.16, pp.123-131, 2003.

[4] M.Saetta , G.Turato , S. Baraldo et al,.” Goblet cell hyperplasia and epithelial inflammation in peripheral airways of smokers with both symptoms of chronic bronchitis and chronic airflow limitation", Am J Respir Crit Care Med., vol.161, pp.1016-1021, 2000,Doi.org/10.1164/ajrccm.161.3.9907080

[5] N.K.Dhillon , W.J.Murphy , M.B. Filla et al.," Down modulation of IFN-gamma signaling in alveolar macrophages isolated from smokers", Toxicol Appl Pharmacol , vol.237, pp. 22-28, 2009; .Doi.org/10.1016/j.taap.2009.02.021

[6] K.W.Garey, M. Neuhauser , R.A. Robbins ,L.H. Danziger ,I. Rubinstein , “ Markers of inflammation in exhaled breath condensate of young healthy smokers", Chest, vol.125, pp. 22-26, 2004; .Doi.org/10.1378/chest.125.1.22
[7] D.O.Warner, " Perioperative abstinence from cigarettes: Physiologic and clinical consequences", Anesthesiology, vol 104, pp.356-367, 2006,.Doi.org/10.1097/00000542-200602000-00023

[8] J.R.Utley ,S.A. Leyland ,C.M. Fogarty ,W.P. Smith , E.B.Knight ,G.J. Feldman , E.F.Wilde , “ Smoking is not a predictor of mortality and morbidity following coronary artery bypass grafting", J Card Surg.,vol.11,pp.377-384,

1996,.Doi.org/10.1111/j.1540-8191.1996.tb00067.x

[9] M.Hollenberg ,D.T. Mangano ,W.S. Browner ,M.J. London , J.F.Tubau , I.M.Tateo, " Study of Perioperative Ischemia Research Group: Predictors of postoperative myocardial ischemia in patients undergoing noncardiac surgery", JAMA, vol 268, pp.205-209, 1992.

[10] В.А.Куликов., В.В. Лихванцев , Р.В. Большедворов , « Критические инциденты при современных методах анестезии», Клиническая анестезиология и реаниматологи, Vol. 4, no.5, pp.42-47.

[11В.В.Субботин , А.В. Ситников , Н.Н.Терехова, « Регистрация и анализ критических инцидентов как способ оценки вариантов общей анестезии в амбулаторной хирургической практике», Вестник анестезиологии и реаниматологи, vol.6,no.3, pp.51-57.

[12] Е.А.Казакова, « Внутренний медицинский аудит на основе регистрации критических инцидентов в отделении анестезиологии многопрофильной клиник»,. Дис. ...канд.мед наук.., pp. 106, 2007

[13] Г.П.Тихова, « Четырехпольная таблица частот - Бритва Оккама в мире статистик»,. Регионарная анестезия и лечение боли, vol. 6, no. 3, pp. 69-75,. 2012.

[14] В.Д.Слепушкин ., В.М.Женило , М.Ю.Осканова , М.В.Женило, Мониторинг церебральных функций в анестезиологии и интенсивной терапии: Владикавказ, 2014, pp.202.

[15] А.М.Овезов, Е.Д. Надькина, С.В. Брагина, П.В. Прокошев Мультимодальная органопротекция при лапароскопической холецистэктомии: Москва, 2013, pp.26.

[16] F.Boer, E Olofson., J.G.Bovil, "Pulmonare uptake of sufentanil during and after constant rate infusion", British J. Anaesthesia, vol.76, pp.203-20,. 1996. Doi.org/10.1093/bja/76.2.203

[17] D.P. Vaughan., A.N.Beckett, “The influence of smoking on the intersubject variation in pentazocine elimination”, . British J. Clin. Pharmaco.,vol.3, pp.279283. 1976..DOI:10.1111/j.1365-2125.1976.tb00604.x

[18] P.Rautoma, N.Vartling, " Smoking increases the requirement for rocuroniu". Canadian. J. Anaesthesia, vol. 45, pp.651-654, 1998/ DOI: 10.1007/bf03012095

[19] G.G.Bestaev , V.D.Slepushkin , A.B.Plieva, "The Peculiarities of Myorelaxants Action in Smokers", EC Anaesthesia. Vol.46, no. 4, pp.11-12, 2018.

[20] Г.Г.Бестаев ， В.Д.Слепушкин Миорелаксанты: сегодня, завтра: Владикавказ, 2019, pp. 125 
[21] H.Teiria , P.Rautoma , A.Yli-Hankala, “ Effect of smoking on dose requirements for vecuronium “, British Journal of Anaesthesia, vol.76, pp.154-155, 1996, Doi.org/10.1093/bja/76.1.154
[22] AlparslanTuran, J.Edward, A. Mascha, ".Smoking and Perioperative Outcomes",/ Anesthesiology, vol. 114, pp. 837-844, 2011. Doi.org/10.1097/01.sa.0000399997.47620.b6

\title{
THE FREQGUENCY OF CRITICAL INCIDENTS AT PERIOPERATIVE PERIOD
} IN SMOKING PATIENTS

\author{
V.D. Slepushkin,* M.C. Musaeva, \\ G.G. Bestaev, P. V. Musaeva \\ North-Ossetian State Medical Academy, \\ Vladikavkaz 362025, Russia
}

\begin{abstract}
Keywords: smoking, critical incidents, perioperative period

\section{Introduction}

There are 5.4 million smoking-related deaths worldwide every year [1]. Smoking increases the risk of adverse postoperative outcomes, especially heart and lung complications [2, 3]. Among smokers, pulmonary problems are prevalent, accounting that smoking impairs the mucus transport, provokes the hyperplasia of goblet cells, the hypersecretion of mucus [4], impairs the function of lung macrophages [5], increases a bronchial reactivity by stimulation of airway inflammation [6]. It is well established that smoking contributes to the development of cardiovascular diseases, but the relationship between smoking and perioperative cardiovascular complications remains controversial [7]. Most studies do not clearly identify the preoperative status of smoking as an independent factor of cardiac events after a non-cardiac surgery [2, $8,9]$. One of the ways to detect the influence of smoking on the occurrence of cardiovascular and pulmonary complications in the intraoperative period is to register critical incidents $[10,11]$. A critical incident is defined as an event during the course of an anaesthetic treatment, which, in the absence of intervention, may lead to undesirable consequences [11].
\end{abstract}

A prospective, randomized, open research was performed, including 114 patients who had laparoscopic cholecystectomy: 1 group - non-smokers (57 people), 2 group - smokers (57 people).

Among smokers, both during the operation and within the near postoperative period (up to 120 minutes), the number of critical incidents increases, connected with the following systems: respiratory system, blood circulation system, and, especially, nervous system: motor excitation, fever, muscular tremors, the feeling of air lack.

Research Results allow to recommend to the anesthetist to pay attention to the smoking experience, the quantity of smoked cigarettes per day and to be wary concerning the occurrence of possible critical incidents.

The purpose of the study: to determine the frequency of critical incidents from the cardiovascular and pulmonary systems in smoking and non-smoking patients in the perioperative period.

\section{Materials and methods}

A controlled prospective randomized clinical trial was performed. The randomization plan was generated by the law of random numbers on the site www.randomization.com (seed 7481). We examined 114 patients with chronic calculous cholecystitis who will undergo laparoscopic cholecystectomy under total intravenous anesthesia based on Diprivan.

Inclusion criterion:

- informed consent of the patient to participate in the study;

- indications for laparoscopic cholecystectomy;

- physical status of the ASAI and ASAII class;

- age 38-52 years.

Exclusion criteria:

- refusal of the patient to participate in the study (lack of informed consent);

- physical status of class ASAIII;

- switching to an open operation;

- a history of polyvalent Allergy, diabetes mellitus, decompensated disorders of the cardiopulmonary system.

Patients are divided into two groups: 1-non smokers (57 patients); 2-smokers per day for at least 20 cigarettes for at least 10 years (57 patients). Demographic data, information about the body mass index and physical status of patients are presented in table 1. The duration of anesthesia in both groups ranged from 54-72 minutes.

Table 1.

Demographic data of patients, body mass index and physical status according to the ASA

\begin{tabular}{|c|c|c|c|c|c|c|}
\hline Groups & Quantity & Gender & Age & Body Mass Index & ASA I & ASA II \\
\hline 1 & 46 & M & $45,3 \pm 5,2$ & $25,8 \pm 4,7$ & 39 & 7 \\
\hline & 11 & Ж & $47,4 \pm 5,1$ & $26,8 \pm 4,8$ & 8 & 3 \\
\hline & 45 & M & $44,4+5,6$ & $24,6 \pm 4,5$ & 35 & 10 \\
\hline Total & 12 & Ж & $46,7 \pm 6,1$ & $26,1 \pm 5,0$ & 8 & 4 \\
\hline
\end{tabular}

Note: the data is presented as $\mathrm{M}+\mathrm{m}$. in all cases, $\mathrm{P}>0.05$. 